\title{
Procedure to Define an Accurate Model for Saturation and Cross-Coupling in IPM Machines
}

\author{
A. Di Gerlando, G. M. Foglia, R. Perini
}

\begin{abstract}
IPM machines are characterized by high saturation and cross-coupling effect. In this paper a procedure is proposed to identify an IPM machine model able to taking into account in a simple and accurate manner both the cited effects. The model is based on functions that express the direct links_ $\bar{\psi}_{s d q}\left(\bar{i}_{s d q}\right)$, and the inverse link $\bar{i}_{s d q}\left(\bar{\psi}_{s d q}\right)$, where $\bar{i}_{s d q}$ and $\bar{\psi}_{s d q}$ are the stator current and stator flux linkage space vectors respectively. The paper shows that such a model is helpful for the analysis of both steady state and transient operation.
\end{abstract}

Index Terms - space vector, saturation, cross coupling, FEM identification of flux linkage-current links, flux linkage-current space vectors links.

\section{INTRODUCTION}

U SUALLY in electrical machines models, the flux linkages-current relations are based on axis inductances $L_{d}, L_{q}$. For their geometrical structure, the IPM (Internal Permanent Magnet) machines are the most prone to high saturation, and great cross-coupling effects. Thus, axis inductances are not constant, but depend on currents, and both $L_{d}, L_{q}$ depend on both currents $i_{d}, i_{q}$. This makes difficult the definition and the usage of functions $L_{d}\left(i_{d}, i_{q}\right)$, $L_{q}\left(i_{d}, i_{q}\right)$, especially if in the state equations the current is chosen as state variable. Therefore, several proposals can be found in literature about how defining inductance functions.

The most used approach is defining some "self and mutual axis inductance functions", given by the partial derivatives of the axis fluxes with respect to the axis currents: in particular, $L_{d d}=\partial \psi_{d}\left(i_{d}, i_{q}\right) / \partial i_{d}, L_{q q}=\partial \psi_{q}\left(i_{d}, i_{q}\right) / \partial i_{q}, L_{d q}=\partial \psi_{d}\left(i_{d}, i_{q}\right) / \partial i_{q}$, $L_{q d}=\partial \psi_{q}\left(i_{d}, i_{q}\right) / \partial i_{d}[1]-[3],[6],[8]$.

Such inductances are then multiplied by the axis current time derivatives, to get the flux linkages derivatives. Functions $\psi_{d}\left(i_{d}, i_{q}\right)$ and $\psi_{q}\left(i_{d}, i_{q}\right)$ can be identified by FEM [1]-[3], [5], [6], [8] or also by test measurements [1], [2]. The FEM or experimental data can be used as look-up tables, or can be used in fitting functions [1], [2], [5], [6], [8]. Anyway, often the formulation is not easy, and sometimes not clear.

An alternative approach is using the flux linkage as state variable: in this case, inductances are not needed, and the links $\bar{\psi}_{s d q}\left(\bar{i}_{s d q}\right)$ and $\bar{i}_{s d q}\left(\bar{\psi}_{s d q}\right)$ are used. This approach is little used in literature [5], [7].

In this paper, an approach similar to [7] is used; differently from to [7], here the aim is to outline in detail the procedure

Lombardy Region Government, Italy, has partially funded the research with POR/FESR INPROVES grant (ID 241363).

A. Di Gerlando, G. M. Foglia and R. Perini are with Politecnico di Milano, Department of Energy, Italy, antonino.digerlando@polimi.it, gianmaria.foglia@polimi.it, roberto.perini@polimi.it. used to define the model; moreover, the model is applied to analyze both steady state and transient operating conditions.

The procedure starts with the definition of the direct link $\bar{\psi}_{s d q}\left(\bar{i}_{s d q}\right)$, and of the inverse link $\bar{i}_{s d q}\left(\bar{\psi}_{s d q}\right)$, where $\bar{i}_{s d q}$ and $\bar{\psi}_{s d q}$ are the stator current and stator flux linkage space vectors respectively. These links are obtained starting from a matrix of points (gained by FEM 2D simulations), which gives the flux linkages values for different values of the current, and for several rotor positions within one pole pair rotor rotation. Even if special attention must be paid in choosing the number of the FEM solution points, in principle FEM approach allows to account for saturation, cross coupling, and PM flux in a very precise manner.

Then, by suited interpolations, continuous functions $\bar{\psi}_{s d q}\left(\bar{i}_{s d q}\right)$ are created. By using a zeroing procedure, a matrix follows which expresses the inverse link $\bar{i}_{s d q}\left(\bar{\psi}_{s d q}\right)$; again, interpolations lead to obtain continuous functions.

A very important step is the identification of the correct definition domain of axis fluxes $\psi_{s d}$ and $\psi_{s q}$, otherwise the extrapolation can give non sensible values; the matter is solved by defining a suited ellipse in the plane $\left(\psi_{s d}, \psi_{s q}\right)$.

Inverse function $\bar{i}_{s d q}\left(\bar{\psi}_{s d q}\right)$ allows using the flux linkage as state variable, so inductances are not needed, and the machine model usage is much simpler.

In the following, the cited steps are presented and described. Then, the use of the model is exemplified in two conditions: 1) evaluation of the MTPA (Maximum Torque Per Ampere) current angle, that is the angle of the current space vector, required for MTPA operation; 2) an example of transient operation with a load torque step variation. In both cases, the proposed model is validated by comparison with 2D FEM simulations (by Ansys Maxwell). Finally, a comparison with a simplified model neglecting cross coupling is carried out.

\section{CONSIDERED IPM MACHINE}

The analysis is applied to an 8-pole IPM machine, with Vshaped PMs. Due to inverter current limits, the machine is equipped with two three-phase windings: each of them is distributed along half the stator periphery; the two current terns are in phase. Table I reports the machine main data, where torque and power data refer to the whole machine.

Fig. 1 shows a one-pole motor cross section.

TABLE I

MAIN DATA OF THE CONSIDERED IPM MACHINE

\begin{tabular}{l|l}
\hline Rated line-line voltage $V_{n}\left[\mathrm{~V}_{\mathrm{rms}}\right]$ & 190 \\
\hline Rated current $I_{n}\left[\mathrm{~A}_{\mathrm{rms}}\right] ;$ rated power $P_{n}[\mathrm{~kW}]$ & $376 ; 180$ \\
\hline Rated torque $T_{n}[\mathrm{Nm}] ;$ rated speed $N_{n}[\mathrm{rpm}]$ & $324 ; 5300$ \\
\hline Pole pair number $n ;$ PM type & $4 ;$ SmCo \\
\hline Phase resistance $R_{p h}[\mathrm{~m} \Omega] ;$ rotor inertia $\left[\mathrm{kgm}^{2}\right]$ & $16.82 ; 0.035$ \\
\hline
\end{tabular}




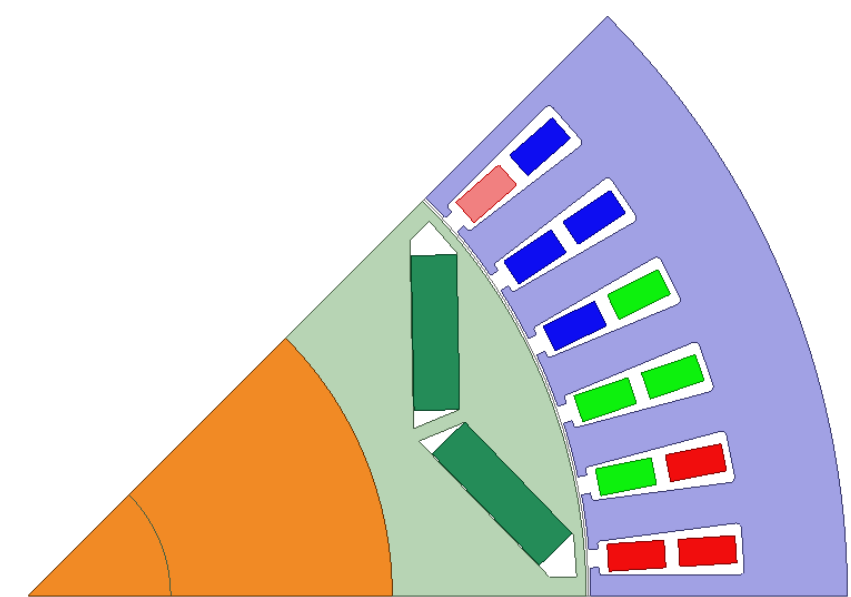

Fig. 1. One-pole cross section of the studied IPM machine.

As cited before, IPM motors are chosen here since saturation and cross-coupling effects are more apparent.

\section{EVALUATION OF DIRECT FLUX - CURRENT RELATIONS}

Let us assume the conventions shown in Fig. 2: stator reference frame $\alpha \beta$, with $\alpha$ axis aligned with stator phase $A$ axis; rotor reference frame $d q$, with $d$ axis aligned with the PM axis; $\theta$ is the electrical angle between $d$ and $\alpha$; stator flux linkage space vector $\bar{\psi}_{s d q}=\psi_{s d}+\mathrm{j} \cdot \psi_{s q}=\Psi_{s d q} \cdot \exp \left(\mathrm{j} \cdot \varphi_{\psi}\right)$; stator current space vector $\bar{i}_{s d q}=i_{s d}+\mathrm{j} \cdot i_{s q}=I_{s d q} \cdot \exp \left(\mathrm{j} \cdot \varphi_{\mathrm{I}}\right)$; both $\bar{\psi}_{s d q}$ and $\bar{i}_{s d q}$ are defined in $d q$ reference frame.

The aim is to obtain the direct relations $\psi_{s d}\left(i_{s d}, i_{s q}\right)$ and $\psi_{s q}\left(i_{s d}, i_{s q}\right)$, which express $\bar{\psi}_{s d q}$ components as a function of $\bar{i}_{s d q}$ components.
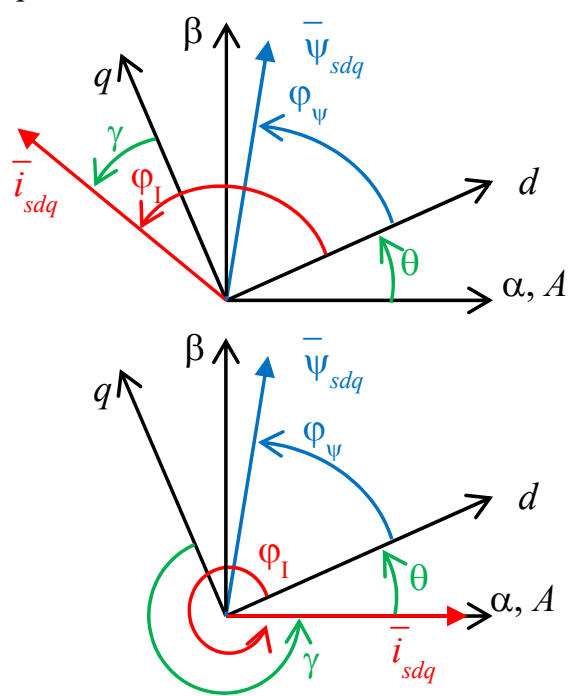

Fig.2. Stator reference frame $\alpha \beta$, rotor reference frame $d q$, current and flux space vectors; top: generic condition; bottom: condition chosen for FEM simulations, with frozen stator currents ( $\bar{i}_{s d q}$ oriented along A phase axis).

To do so, we should set a rotor position, and move $\bar{i}_{s d q}$ to span a double polar pitch (360 electrical deg); each position corresponds to a couple $\left(i_{s d}, i_{s q}\right)$, and for each couple the flux values $\psi_{s d}, \psi_{s q}$ are evaluated. The same result is obtained if $\bar{i}_{s d q}$ is fixed in $\alpha \beta$ reference frame, and the rotor is rotated. For the FEM simulations, the latter method is much simpler (because $\bar{i}_{s d q}$ fixed in $\alpha \beta$ requires constant values of the phase currents): thus, several 2D FEM simulations are performed, where we set $\bar{i}_{s d q}$, and we consider $k_{p o s}$ rotor positions within a double polar pitch (i.e., $\theta$ assumes $k_{p o s}$ values $\theta_{k}$ between 0 and 360 electrical deg). This can be done either by using a magneto-static solver, and a script file which changes the rotor position, or by a transient solver, which moves the rotor. Vector $\bar{i}_{s d q}$ can be placed everywhere in $\alpha \beta$ frame; a sensible choice is aligning it with $\alpha$ axis (Fig. 2 (bottom)), that is $\varphi_{\mathrm{I}}=-\theta$, thus $\bar{i}_{s d q}=I_{s d q} \cdot \exp (-\mathrm{j} \cdot \theta)$, or $i_{s d}=$ $I_{s d q} \cdot \cos (-\theta), \quad i_{s q}=I_{s d q} \cdot \sin (-\theta)$. This choice does not correspond to any motor operating condition, but it is the simplest one for the FEM simulations.

Moreover, the instantaneous values of the phase currents equal $i_{A}=\sqrt{ }(2 / 3) \cdot \mathrm{I}_{s d q}, i_{B}=i_{C}=-i_{A} / 2$.

For each rotor position $\theta_{k}$, the FEM solver gives the stator phase flux linkages $\psi_{s A}, \psi_{s B}, \psi_{s C}$, and $\bar{\psi}_{s d q}$ is evaluated by applying the space vector definition

$$
\begin{gathered}
\bar{\psi}_{s d q}=\psi_{s d}+j \psi_{s q}=\sqrt{2 / 3} \cdot\left\{\psi_{s A}+\bar{a} \cdot \psi_{s B}+\bar{a}^{2} \cdot \psi_{s C}\right\} \\
\cdot \exp (-j \cdot \theta), \quad \bar{a}=\exp (j 2 \pi / 3)
\end{gathered}
$$

Thus, for one value $I_{s d q}$ (magnitude of $\bar{i}_{s d q}$ ), $\psi_{s d}$ and $\psi_{s q}$ are expressed as a function of $\varphi_{I}=-\theta$ (phase of $\bar{i}_{s d q}$ ).

Actually, there is not a continuous function, but two vectors $\left[\psi_{s d}\right]$ and $\left[\psi_{s q}\right]$, with $k_{p o s}$ rows. To obtain a continuous function, FFT is performed on the vectors $\left[\psi_{s d}\right]$ and $\left[\psi_{s q}\right]$, that leads to the following Fourier development:

$$
\psi_{s d}(\theta)=\sum_{h} \Psi_{s d h} \cos (h \theta), \psi_{s q}(\theta)=\sum_{h} \Psi_{s q h} \sin (h \theta)(2)
$$

with $h=1 \ldots h_{M A X}$ harmonic order, and $\Psi_{s d h}, \Psi_{s q h}$ the FFT coefficients; do note that, thanks to the machine symmetry, $\psi_{s d}(\theta)$ has only cosine terms, and $\psi_{s q}(\theta)$ only sine terms.

Now, the procedure is repeated for $k_{I}$ values of $I_{s d q}$, between 0 and the maximum current $I_{s d q_{-} \max }$ (here equal to $1000 \mathrm{~A}$ ). Thus, we obtain 3 vectors with $k_{I}$ rows: a current vector $\left[I_{s d q}\right]$ and two function vectors $\left[\psi_{s d}(\theta)\right]$ and $\left[\psi_{s q}(\theta)\right]$, each row corresponding to one $I_{s d q}$ value. Actually, the function vectors $\left[\psi_{s d}(\theta)\right]$ and $\left[\psi_{s q}(\theta)\right]$ are represented by the corresponding FFT coefficient vectors; thus, we have $h_{M A X}$ vectors [ $\left.\Psi_{s d h}\right]$, and $h_{M A X}$ vectors [ $\Psi_{s q h}$ ] (one vector for each harmonic; each vector has $k_{I}$ rows, one row for each $I_{s d q}$ value). To obtain a continuous function of the current, an interpolation on the FFT coefficients vectors is applied; in particular, for each harmonic $h$, a continuous function is obtained by interpolating $\left[\Psi_{s d h}\right],\left[\Psi_{s q h}\right]$, with respect to $\left[I_{s d q}\right]$ :

$$
\begin{aligned}
& \Psi_{s d h}\left(I_{s d q}\right)=\operatorname{interp}\left(\left[\Psi_{s d h}\right],\left[I_{s d q}\right]\right), \\
& \Psi_{s q h}\left(I_{s d q}\right)=i n t \operatorname{erp}\left(\left[\Psi_{s q h}\right],\left[I_{s d q}\right]\right)
\end{aligned}
$$

These functions replace the FFT coefficients $\Psi_{s d h}, \Psi_{s q h}$ in (2). Moreover, the relation $\varphi_{I}=-\theta$ is applied. Thus, the fluxes $\psi_{s d}$ and $\psi_{s q}$ are obtained as continuous functions of both $I_{s d q}$ and $\varphi_{I}$ (magnitude and phase of $\bar{i}_{s d q}$ ):

$$
\begin{aligned}
& \psi_{s d}\left(I_{s d q}, \varphi_{I}\right)=\sum_{h} \psi_{s d h}\left(I_{s d q}\right) \cdot \cos \left(-h \cdot \varphi_{I}\right) \\
& \psi_{s q}\left(I_{s d q}, \varphi_{I}\right)=\sum_{h} \psi_{s q h}\left(I_{s d q}\right) \cdot \sin \left(-h \cdot \varphi_{I}\right)
\end{aligned}
$$


Some examples of $\psi_{s d}\left(I_{s d q}, \theta\right), \psi_{s q}\left(I_{s d q}, \theta\right)$, are shown in Fig. 3 .
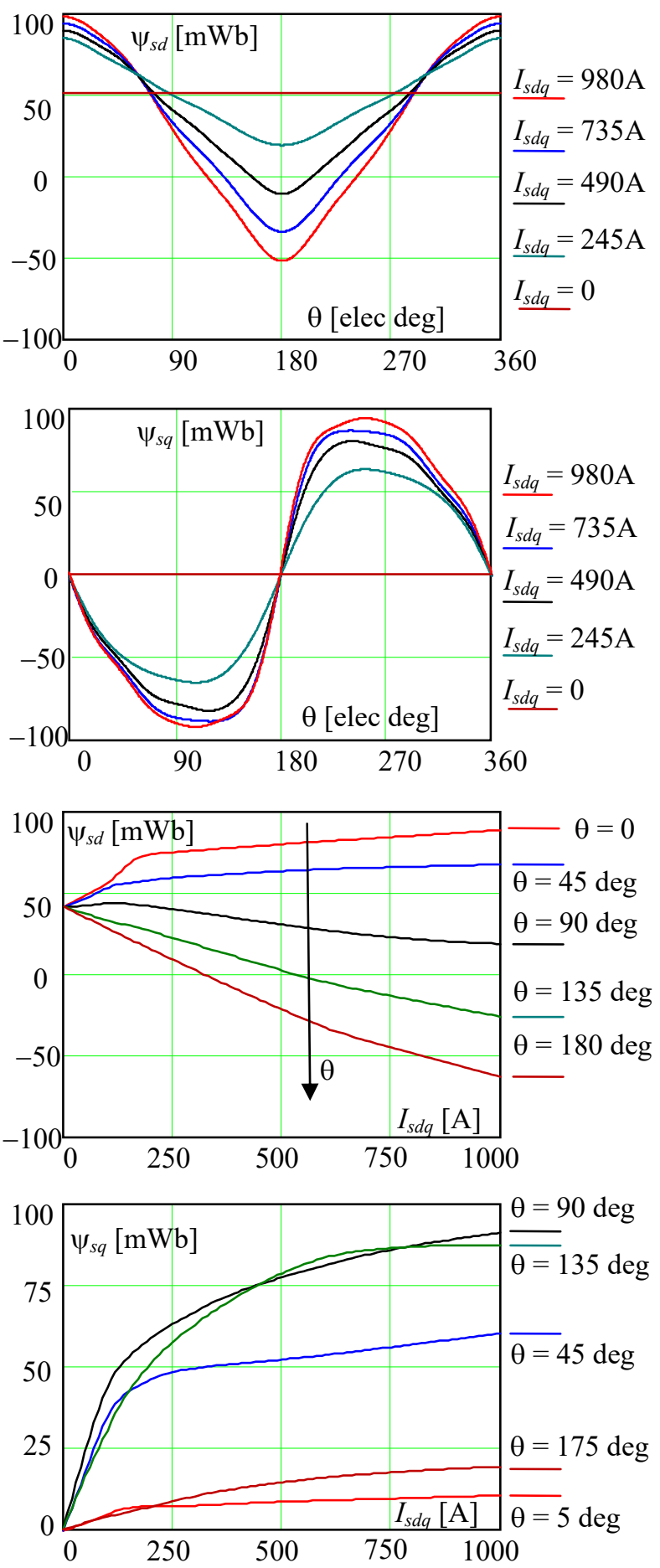

Fig.3. Examples of functions $\psi_{s d}\left(I_{s d q}, \theta\right), \psi_{s q}\left(I_{s d q}, \theta\right)$.

Considering the relations between $I_{s d q}, \varphi_{I}$ and $i_{s d}, i_{s q}$

$$
I_{s d q}=\sqrt{i_{s d}^{2}+i_{s q}^{2}} \varphi_{I}=\operatorname{atan}\left(i_{s q} / i_{s d}\right)
$$

$\psi_{s d}$ and $\psi_{s q}$ are expressed as continuous functions of $i_{s d}, i_{s q}$ :

$$
\begin{gathered}
\psi_{s d}\left(i_{s d}, i_{s q}\right)= \\
=\sum_{h=1}^{h_{M A X}} \Psi_{s d h}\left(\sqrt{\left.{i_{s d}{ }^{2}+i_{s q}{ }^{2}}^{2}\right) \cdot \cos \left(-h \cdot \operatorname{atan}\left(\frac{i_{s q}}{i_{s d}}\right)\right)}\right.
\end{gathered}
$$

$$
\begin{gathered}
\psi_{s q}\left(i_{s d}, i_{s q}\right)=\sum_{h=1}^{h=h_{M A X}} \psi_{s q h}\left(\sqrt{i_{s d}^{2}+i_{s q}^{2}}\right) . \\
\cdot \sin \left(-h \cdot \operatorname{atan}\left(i_{s q} / i_{s d}\right)\right)
\end{gathered}
$$

Thus, the space vector of the stator flux linkages $\bar{\psi}_{s d q}$ is completely defined, as a function either of $I_{s d q}, \varphi_{\mathrm{I}}$ or $i_{s d}, i_{s q}$.

$$
\begin{aligned}
& \overline{\Psi_{s d q}}\left(I_{s d q}, \varphi_{I}\right)=\psi_{s d}\left(I_{s d q}, \varphi_{I}\right)+j \cdot \psi_{s q}\left(I_{s d q}, \varphi_{I}\right) \\
& \overline{\Psi_{s d q}}\left(i_{s d}, i_{s q}\right)=\psi_{s d}\left(i_{s d}, i_{s q}\right)+j \cdot \psi_{s q}\left(i_{s d}, i_{s q}\right)
\end{aligned}
$$

As concerns the values of $k_{p o s}, k_{l}, h_{M A X}$, the following remarks hold:

$-k_{\text {pos }}$ should be rather high, to allow a good reconstruction of the flux-angle relation; we adopted $k_{\text {pos }}=1080$;

$-k_{I}$ cannot be too low, otherwise a wrong flux-current relation is obtained; we adopted $k_{I}=40$;

- as regards the maximum order $h_{M A X}$, we assumed $h_{M A X}=7$, in order to filter the tooth harmonics effect. An example of the amplitude of such harmonics is shown later, in Fig. 5.

\section{EVALUATION OF INVERSE CURRENT-FLUX RELATIONS}

In order to adopt the flux as a state variable, the inverse relations which express $i_{s d}, i_{s q}$ as a function of $\psi_{s d}, \psi_{s q}$ are needed. For a couple of values $\psi_{s d}{ }^{*}, \psi_{s q}{ }^{*}$, the corresponding values $i_{s d}{ }^{*}, i_{s q}{ }^{*}$ can be found by solving numerically the system (6); thus, $i_{s d}{ }^{*}, i_{s q}{ }^{*}$ are the solutions of the system:

$$
\left\{\begin{array}{l}
\psi_{s d}\left(i_{s d}{ }^{*}, i_{s q}{ }^{*}\right)=\psi_{s d}{ }^{*} \\
\psi_{s q}\left(i_{s d}{ }^{*}, i_{s q}{ }^{*}\right)=\psi_{s q}{ }^{*}
\end{array}\right.
$$

By solving (9) for some values of $\psi_{s d}$ and $\psi_{s q}$, corresponding $i_{\text {sd }}, i_{s q}$ values of the relations $i_{s d}\left(\psi_{s d}, \psi_{s q}\right), i_{s q}\left(\psi_{s d}, \psi_{s q}\right)$ follow. In particular, $N_{d}$ values of $\psi_{s d}$ and $N_{q}$ values of $\psi_{s q}$ are chosen, equally spread between maximum and minimum values of $\psi_{s d}$ and $\psi_{s q}$; for instance, Fig. 3 shows that the ranges are about $-50 \mathrm{mWb}<\psi_{s d}<100 \mathrm{mWb},-100 \mathrm{mWb}<$ $\psi_{s q}<100 \mathrm{mWb}$. Thus, two vectors are obtained, [ $\left.\psi_{s d_{-} i}\right]$ of $N_{d}$ rows, $\left[\psi_{s q_{i}}\right]$ of $N_{q}$ rows (footer " $i$ " stands for "inverse function"). By solving (9) for all the values of vectors $\left[\psi_{s d_{-} i}\right]$, $\left[\psi_{s q_{-} i}\right]$, two matrices are obtained, $\left[i_{s d_{-} i}\right],\left[i_{s q_{-} i}\right]$, both with $N_{d}$ rows and $N_{q}$ columns; each element $(j, k)$ satisfies system (9):

$$
\left\{\begin{array} { l } 
{ \Psi _ { s d } ( [ i _ { s d _ { - } i } ] _ { j , k ^ { \prime } } [ i _ { s q _ { - } i } ] _ { j , k } ) = [ \Psi _ { s d } ] _ { j } } \\
{ \Psi _ { s q } ( [ i _ { s d _ { - } i } ] _ { j , k ^ { \prime } } [ i _ { s q _ { - } i } ] _ { j , k } ) = [ \Psi _ { s q } ] _ { k } }
\end{array} \quad \left\{\begin{array}{l}
j=1 . . N_{d} \\
k=1 . . N_{q}
\end{array}\right.\right.
$$

Next step is to move from matrixes $\left[i_{s d_{-} i}\right],\left[i_{s q_{i}}\right]$ to continuous functions $i_{s d}\left(\psi_{s d}, \psi_{s q}\right), i_{s q}\left(\psi_{s d}, \psi_{s q}\right)$. To do so, a double interpolation of matrixes $\left[i_{s d_{-}}\right],\left[i_{s q_{-} i}\right]$ is performed: first, each of the $N_{q}$ columns $\left[i_{s d_{-} i}\right]_{c o l} k,\left[i_{s q_{-}}\right]_{\text {col_k }}$ (each column has $N_{d}$ rows) are interpolated with $\left[\psi_{s d_{-} i}\right]$ (which has $N_{d}$ rows); we obtain $N_{q}$ interpolating functions; thereafter, these $N_{q}$ functions are interpolated with $\left[\psi_{s q_{-}}\right]$(which has $N_{q}$ rows).

$$
\begin{gathered}
i_{s d_{\text {interp }}}\left(\psi_{s d}, \psi_{s q}\right)= \\
=\operatorname{interp}\left(\operatorname{interp}\left(\left[i_{s d_{-} i}\right]_{\text {col_k }},\left[\psi_{s d_{-} i}\right]\right),\left[\psi_{s q_{-} i}\right]\right)
\end{gathered}
$$




$$
\begin{gathered}
i_{s q_{\text {interp }}}\left(\psi_{s d}, \psi_{s q}\right)= \\
=\operatorname{interp}\left(\operatorname{interp}\left(\left[i_{s q_{-} i}\right]_{\text {col_k }},\left[\psi_{s d_{-} i}\right]\right),\left[\psi_{s q_{-} i}\right]\right)
\end{gathered}
$$

\section{DEFINITION OF THE CORRECT FLUX RANGES}

In functions (4), the $\bar{i}_{s d q}$ domain is $0<I_{s d q}<I_{\text {sdq_max }}, 0<\varphi_{I}$ $<2 \pi$ : in $\left(i_{s d}, i_{s q}\right)$ plane, the current limiting line is a circumference with radius equal to $I_{s d q_{-} \max }$. In $\left(\psi_{s q}, \psi_{s d}\right)$ plane, the corresponding limiting flux contour is obtained by plotting functions (4) in a polar plot, at the maximum current $I_{\text {sdq_max }}$; a line with a rough elliptic shape is found (black continuous line in Fig. 4). Points outside this rough elliptic line correspond to values of current outside the definition domain. This implies that the currents $i_{s d}{ }^{*}, i_{s q}{ }^{*}$ found by (9) are meaningful only if the fluxes $\psi_{s d}{ }^{*}, \psi_{s q}{ }^{*}$ are inside the cited rough elliptic line.

In (11), the domain of definition is a rectangle with $\psi_{s d_{\text {min }}}<$ $\psi_{s d}<\psi_{s d_{\_} \max }, \psi_{s q_{-} \min }<\psi_{s q}<\psi_{s q_{-} \max }$ (green lines in Fig. 4). The corners of this rectangle are outside the rough elliptic contour, therefore the definition domain of (11) is not correct; indeed, (11) do not work well (some current values get very high). A correction must be introduced in (11), to make the fluxes stay inside the rough elliptic line.

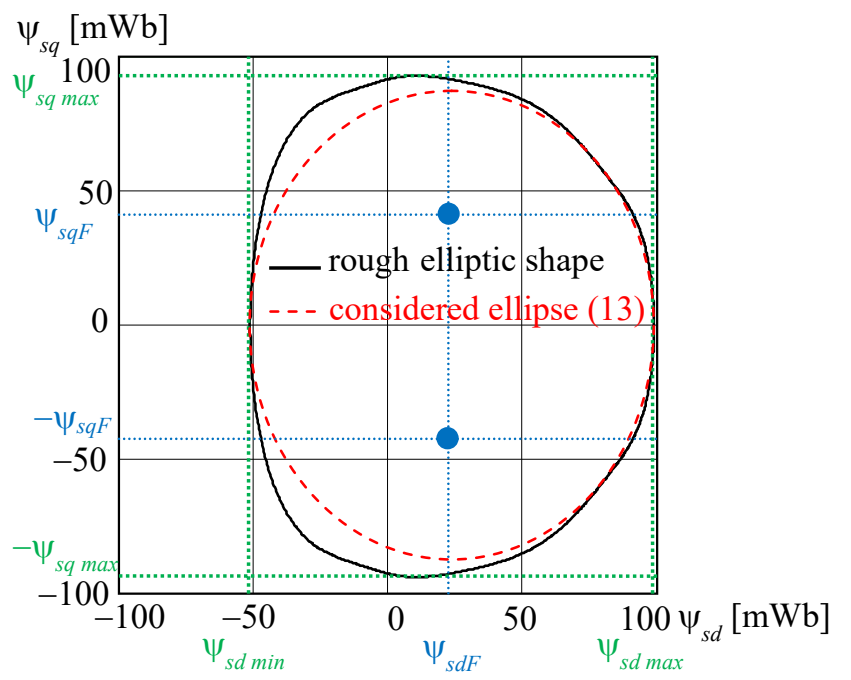

Fig. 4. Original elliptic shape, corresponding to polar plot of (4), and ellipse considered in (13).

First, the rough elliptic contour is substituted by an ellipse (red dotted line in Fig. 4) which is as much as possible similar to the rough elliptic contour, and completely inside it. By means of geometrical relations, both the foci coordinates $\left(\psi_{s d F}, \pm \psi_{s q F}\right)$ and the ellipse characteristic constant FD (Foci Distance, i.e. the sum of the distances from the ellipse foci) can be expressed as a function of the flux limiting values $\left(\psi_{s d_{-} \min }, \psi_{s d_{-} \max }, \psi_{s q_{\_} \min }, \psi_{s q_{-} \max }\right.$, with $\left.\left|\psi_{s q_{-} \min }\right|=\left|\psi_{s q_{-} \max }\right|\right)$.

Then, the sum of the distances of a generic point $\left(\psi_{s d}, \psi_{s q}\right)$ from the foci $\left(\psi_{s d F},-\psi_{s q F}\right)$ and $\left(\psi_{s d F}, \psi_{s q F}\right)$ equals

$$
f d\left(\psi_{s d}, \psi_{s q}\right)=\sqrt{\left(\psi_{s d}-\psi_{s d F}\right)^{2}+\left(\psi_{s q}-\psi_{s q F}\right)^{2}}+
$$

$$
+\sqrt{\left(\psi_{s d}-\psi_{s d F}\right)^{2}+\left(\psi_{s q}+\psi_{s q F}\right)^{2}}
$$

and the condition to make the fluxes stay inside the ellipse is simply $f d\left(\psi_{s d}, \psi_{s q}\right)<F D=2 \cdot \psi_{s q_{-} \max }$.

Thus, (11) changes in (13): a zero current value is forced in case of flux values outside the correct definition domain:

$$
\begin{gathered}
\mathrm{i}_{s d}\left(\psi_{s d}, \psi_{s q}\right)= \\
=\text { if }\left[f d\left(\psi_{s d}, \psi_{s q}\right)<F D, \mathrm{i}_{\text {sd_interp }}\left(\psi_{s d}, \psi_{s q}\right), 0\right] \\
\mathrm{i}_{s q}\left(\psi_{s d}, \psi_{s q}\right)= \\
=\text { if }\left[f d\left(\psi_{s d}, \psi_{s q}\right)<F D, \mathrm{i}_{s q_{-} \text {interp }}\left(\psi_{s d}, \psi_{s q}\right), 0\right]
\end{gathered}
$$

By considering the space vectors, the inverse relations current-flux can be defined as

$$
\begin{aligned}
\overline{l_{l n v}}\left(\bar{\psi}_{s d q}\right)= & i_{s d}\left(\operatorname{Re}\left(\bar{\psi}_{s d q}\right), \operatorname{Im}\left(\bar{\psi}_{s d q}\right)\right) \\
& +j i_{s q}\left(\operatorname{Re}\left(\bar{\psi}_{s d q}\right), \operatorname{Im}\left(\bar{\Psi}_{s d q}\right)\right)
\end{aligned}
$$

\section{MODEL SUMMARY AND TORQUE EXPRESSION}

At the end, the model is based just on some interpolating functions, and some vectors or matrices:

- the direct relations flux - current (4) are obtained by means of interpolating functions (3), based on vectors $\left[\Psi_{s d h}\right]$, $\left[\Psi_{s q h}\right],\left[I_{s d q}\right]$ (gained by processing the data of some FEM simulations);

- the inverse relations current - flux (13) are obtained by means of interpolating functions (11), based on matrices $\left[i_{s d_{-} i}\right],\left[i_{s q_{-} i}\right]$ (gained by numerical solution of system (9) ).

Both the FEM simulations and the numerical solution of system (9) are performed just once, in order to get the vectors or matrices needed for the interpolation. Afterwards, the model just requires a software able to perform the interpolations (in our application, we adopted Mathcad 11). The electromagnetic torque $T_{e}$ has the classical expression:

$$
T_{e}=-2 \cdot n \cdot \operatorname{Im}\left(\bar{\psi}_{s d q} \underline{\mathrm{i}}_{s d q}\right),
$$

where 2 is due to the two three-phase windings, $n$ is the polepair number, $\operatorname{Im}()$ is the Imaginary part operator, $\underline{i}_{s d q}$ is the conjugate of $\bar{i}_{s d q}$.

$\bar{\psi}_{s d q}$ (and, thus, $T_{e}$ too) can be expressed as a function of either $i_{s d}$ and $i_{s q}$ or $I_{s d q}$ and $\varphi_{I}$, by means of (8) or (7) respectively.

\section{EVALUATION OF THE CURRENT ANGLE FOR MTPA}

If $T_{e}$ is expressed as a function of $\mathrm{I}_{\mathrm{sdq}}$ and $\varphi_{\mathrm{I}}$, the current angle $\varphi_{\text {I_MTPA, }}$, which gives the MTPA condition, can be obtained by performing a numerical derivative of the function $T_{e}\left(I_{s d q}, \varphi_{I}\right)$ with respect to $\varphi_{I}$, and by zeroing such derivative. In such calculation, some troubles may occur, because $\varphi_{I_{-} M T P A}$ is higher than $90 \mathrm{deg}$; the matter is solved by substituting $\varphi_{I}$ with $\gamma+90 \mathrm{deg}$ (see Fig. 2), and performing the same operation by using angle $\gamma$. As a result, the 
proposed model gives angle $\gamma_{\text {MTPA_mod }}$ (which gives the MTPA condition), as a function of $I_{s d q}$ (magnitude of $\bar{i}_{s d q}$ ):

$$
\begin{gathered}
T_{e}\left(I_{s d q}, \varphi_{I}\right)=-2 n \cdot \operatorname{Im}\left\{\overline{\Psi_{s d q}}\left(I_{s d q}, \varphi_{I}\right) \cdot I_{s d q} \cdot e^{-j \varphi_{I}}\right\} \\
T_{e}\left(I_{s d q}, \gamma\right)=-2 \cdot n \cdot \operatorname{Im}\left\{\overline{\Psi_{s d q}}\left(I_{s d q}, \gamma+\pi / 2\right)\right. \\
\left.\left[I_{s d q} \cdot \exp (-j(\gamma+\pi / 2))\right]\right\} \\
\frac{\Delta T_{e}\left(I_{s d q}, \gamma\right)}{\Delta \gamma}=\frac{T_{e}\left(I_{s d q}, \gamma+\Delta \gamma\right)-T_{e}\left(I_{s d q}, \gamma\right)}{\Delta \gamma} \\
\gamma_{\text {MTPA_mod }}\left(I_{s d q}\right)=\text { zeroing }\left[\frac{\Delta T_{e}\left(I_{s d q}, \gamma\right)}{\Delta \gamma}, \gamma\right]
\end{gathered}
$$

In order to validate this procedure, the angle $\gamma_{M T P A}$ has been found also by FEM simulations. In particular, the same solutions (with fixed current and moving rotor), which gave the flux linkages, also gave the holding torque (which is defined just as the torque with fixed current and moving rotor): therefore, for each of the $k_{I}$ current values, we have a vector $\left[H T_{k l}\right]$, of $k_{p o s}$ holding torque values. In principle, $\left[H T_{k I}\right]$ can be transformed in a continuous function $H T_{k l}(\theta)$ of the rotor position $\theta$, by means of an spline function; then, $H T_{k l}(\theta)$ can be derived, to gain the $\theta_{\text {MTPA }}$ value which maximizes the torque. Actually, the procedure is not so straightforward, due to stator slotting and harmonic MMFs, which cause a ripple in the holding torque. Thus, before derivation, function $H T_{k l}(\theta)$ is to be filtered to eliminate tooth harmonics; a simple way is performing a Fourier development of $H T_{k l}(\theta)$, and reconstructing the function just with the low order harmonics. Fig. 5 shows an example of $H T_{k l}(\theta)$ function, and the corresponding filtered function $H T_{k I}$ filt $(\theta)$, for $I_{s d q}=602 \mathrm{~A}$.

According to Fig.2(b), the $\theta-\gamma$ relation is $\gamma+\theta=\pi \cdot 3 / 2$. Thus, $\gamma_{\text {MTPA_mod }}$ can be found by differentiating $H T_{k I}$ filt $(\pi \cdot 3 / 2$ $-\gamma$ ) with respect to $\gamma$, and by zeroing the derivative.

Fig. 6 shows the comparison between $\gamma_{M T P A_{-} \bmod }\left(I_{\text {sdq }}\right)$ and $\gamma_{M T P A_{-} F E M}$ : the good agreement validates the proposed model.

\section{ANALYSIS OF STEADY STATE OPERATION IN MTPA}

In MTPA operation, angle $\gamma$ value is obtained by (19). Therefore, given a desired torque value $T_{e}^{*}$, the required current space vector $I_{s d q} * \cdot \exp \left[\mathrm{j}\left(\gamma_{M T P A} *+\pi / 2\right)\right]$ is obtained by (19) and (17):

$$
T_{e}\left(I_{s d q}{ }^{*}, \gamma_{M T P A}{ }^{*}\right)=T_{e}^{*}, \gamma_{M T P A}{ }^{*}=\gamma_{M T P A_{-} \bmod }\left(I_{s d q}{ }^{*}\right)
$$

By solving numerically (20), the required value $I_{s d q}{ }^{*}$ and $\gamma_{M T P A}{ }^{*}$ are obtained. Then, the required voltage space vector $V_{s d q}{ }^{*} \cdot \exp \left[\mathrm{j} \varphi_{V}{ }^{*}\right]$ is gained by the voltage law:

$$
\begin{array}{r}
V_{s d q}{ }^{*} \cdot \exp \left(\mathrm{j} \varphi_{V}{ }^{*}\right)=R_{p h} \cdot I_{s d q}{ }^{*} \exp \left(j\left(\gamma_{M T P A}{ }^{*}+\pi / 2\right)\right)+ \\
j \omega \bar{\Psi}_{s d q}\left(I_{s d q}{ }^{*}, \gamma_{M T P A}{ }^{*}+\pi / 2\right)
\end{array}
$$

where $\omega$ is the angular frequency $(\omega=n 2 \pi N / 60 ; N$ [rpm] $=$ speed, $n=$ pole pair).

For instance, an operating point in MTPA is as follows:

$$
\begin{gathered}
N_{\text {op }}=5000 \mathrm{rpm} ; \quad T_{\text {e.op }}=260 \mathrm{Nm}: \\
I_{\text {sdq.op }}=504.9 \mathrm{~A} ; \gamma_{\text {MTPA.op }}=46.4 \mathrm{deg} \\
V_{\text {sdq.op }}=172.8 \mathrm{~V} ; \quad \varphi_{\text {V.op }}=170.4 \mathrm{deg}
\end{gathered}
$$

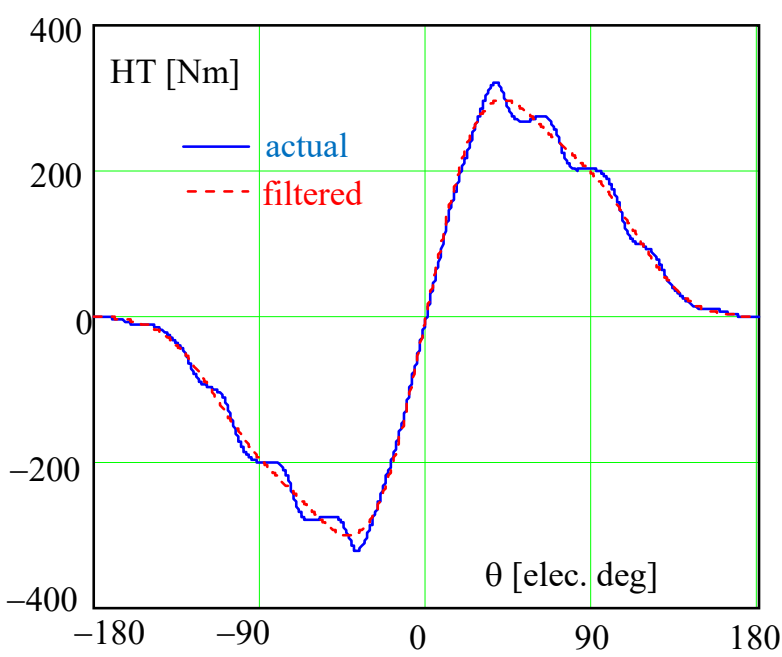

Fig.5. Holding torque as a function of the electrical angle. Continuous line: actual values, with ripple due to stator slotting. Dotted line: filtered function.

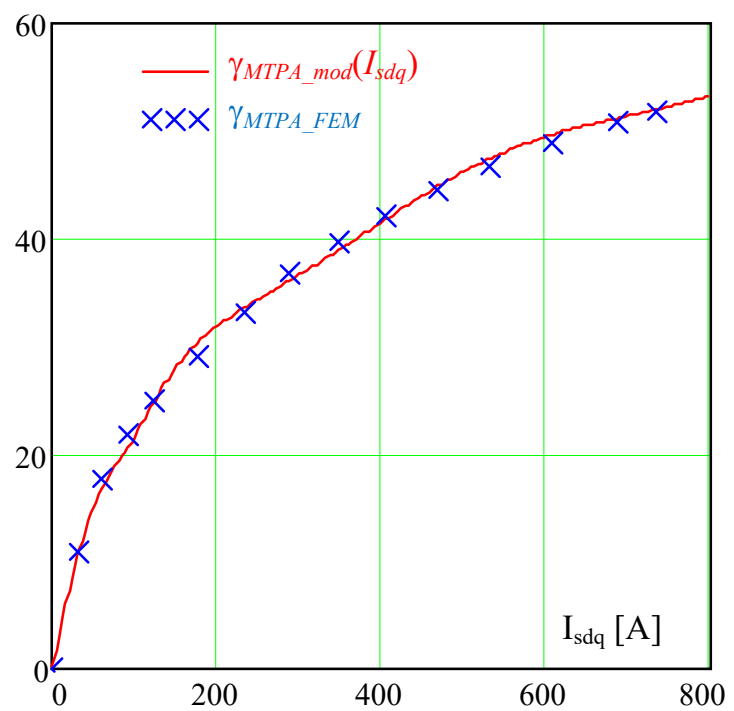

Fig. 6. Current angle $\gamma_{M T P A}$ which gives maximum torque per ampere condition (MTPA), as a function of $I_{s d q}$ (magnitude of current space vector); comparison between the angles evaluated by the proposed model $\left(\gamma_{M T P A_{-} m o d}\left(I_{\text {sdq }}\right)\right)$ and by FEM simulations $\left(\gamma_{M T P A_{-} \text {FEM }}\right)$.

\section{EXAMPLE OF TRANSIENT OPERATION}

In order to validate the proposed model in transient operation, a simple electromechanical transient has been simulated, and compared with a FEM solution. Since the aim is the flux-current link, and not the system stability, an open loop operation is considered, without any control system. In particular, a three-phase sinusoidal supply voltage (with constant rms phase voltage $\mathrm{V}_{\text {sph }}$ and constant angular frequency $\omega$ ) is assumed and, starting from a steady state operation, a step variation of a fluid-dynamic load torque (i.e.: torque $\propto$ speed squared) has been applied.

The phase $A$ voltage expression is $v_{A}(t)=\sqrt{2} \cdot V_{s p h} \cdot \cos \left(\theta_{V}\right)$, where $\theta_{V}=\omega \cdot t+\theta_{V 0}$. The phase voltage space vector in $d q$ reference frame $\bar{v}_{s d q}$ can be expressed as:

$$
\begin{gathered}
\bar{v}_{s d q}=V_{s d q} \cdot \exp \left(\mathrm{j} \varphi_{V}\right)=\sqrt{2 / 3}\left(\sqrt{2} V_{s p h}\right)\left[\cos \left(\theta_{V}\right)+\bar{a} \cdot\right. \\
\left.\cos \left(\theta_{V}-2 \pi / 3\right)+\bar{a}^{2} \cdot \cos \left(\theta_{V}-4 \pi / 3\right)\right] \cdot \exp (-j \theta)
\end{gathered}
$$


where $\varphi_{V}$ is the phase with respect to $d$ axis, $\theta_{V}$ is the phase with respect to real axis $\alpha, \theta$ is the angle between $d$ axis and real axis $\alpha$ (Fig. 7(a)); $\bar{a}=\exp (\mathrm{j} 2 \pi / 3) ; V_{s d q}=\sqrt{3} V_{s p h}$. It is assumed that $t=0$ occurs when $d$ axis is aligned to real axis $\alpha$ (Fig. $7 \mathrm{~b}$ ), thus $\theta_{V 0}=\varphi_{V 0}$; moreover, it is assumed that in $t$ $=0$ the system is at steady state at operating point (22); thus, $\varphi_{V 0}=\varphi_{V . o p}, \omega=\omega_{o p}, V_{s d q}=V_{\text {sdq.op }}$. Thus, $\bar{v}_{s d q}$ depends on time $t$ and on $d$ axis position $\theta$, and $\bar{v}_{s d q}$ becomes:

$$
\begin{array}{r}
\bar{v}_{s d q}(t, \theta)=\sqrt{2 / 3}\left(\sqrt{2 / 3} V_{s d q . o p}\right)\left[\cos \left(\omega_{o p} t+\varphi_{V . o p}\right)+\right. \\
\bar{a} \cdot \cos \left(\omega_{o p} t+\varphi_{V . o p}-2 \pi / 3\right)+\bar{a}^{2} \cdot \cos \left(\omega_{o p} t+\varphi_{V . o p}-\right. \\
4 \pi / 3)] \cdot \exp (-j \theta)
\end{array}
$$
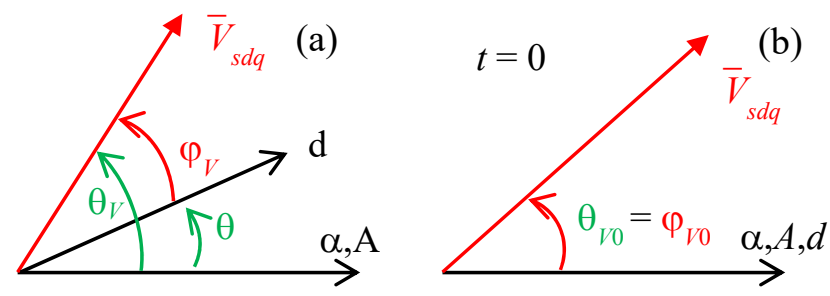

Fig.7. Phase voltage space vector. (a): generic position. (b): initial condition for the transient operation.

It is assumed that the fluid-dynamic load torque $T_{L}$ starts from the steady state value (22) and, after a time interval $\Delta \mathrm{t}_{\mathrm{TL}}$, decreases by $10 \%$. Thus, $\mathrm{T}_{\mathrm{L}}$ depends on the time and on the angular frequency $\omega$, and its expression can be written as:

$$
T_{L}(t, \omega)=i f\left[t<\Delta t_{T L}, T_{o p}, 0.9 \cdot T_{o p} \cdot\left(\omega / \omega_{n}\right)^{2}\right]
$$

with $\omega_{n}$ rated angular frequency $\left(\omega_{n}=2 \pi n \cdot N_{n} / 60=2220 \mathrm{rad} / \mathrm{s}\right)$. The state variables are $\theta, \omega$, and the stator flux linkage space vector $\bar{\psi}_{s d q}$; the current space vector is obtained by the inverse function (14); the state equations of the machine are:

$$
\begin{gathered}
\frac{d \theta}{d t}=\omega \\
\frac{J_{t}}{n} \frac{d \omega}{d t}=-n \cdot \operatorname{Im}\left\{\bar{\Psi}_{s d q} \cdot \underline{i_{i n v}}\left(\bar{\Psi}_{s d q}\right)\right\}-T_{L}(t, \omega) \\
\frac{d \bar{\psi}_{s d q}}{d t}=\bar{v}_{s d q}(t, \theta)-R_{p h} \cdot \overline{i_{i n v}}\left(\bar{\Psi}_{s d q}\right)-j \omega \bar{\psi}_{s d q}
\end{gathered}
$$

System (26) is integrated by backward Euler method, with a time step of $50 \mu \mathrm{s}$. As concerns the FEM simulation, the same transient was implemented in Ansys Maxwell, by means of a 2D transient simulation; the machine model is the one shown in Fig. 1, and a master-slave boundary condition has been used.

Fig.s 8 (a) and (b) show the comparison between the model results and the FEM results as concerns the electromagnetic torque $T_{e}$ and the mechanical speed $N$.

It should be observed that the original FEM electromagnetic torque waveform (actual points) is affected by significant torque ripple (as shown in Fig. 5): it can be eliminated by filtering the original waveform by moving average.

The agreement is fair, and considering that the model simulation takes a few minutes, whereas the FEM simulation takes a few days, the proposed model can be considered a useful tool, especially for machine design and operation analysis.
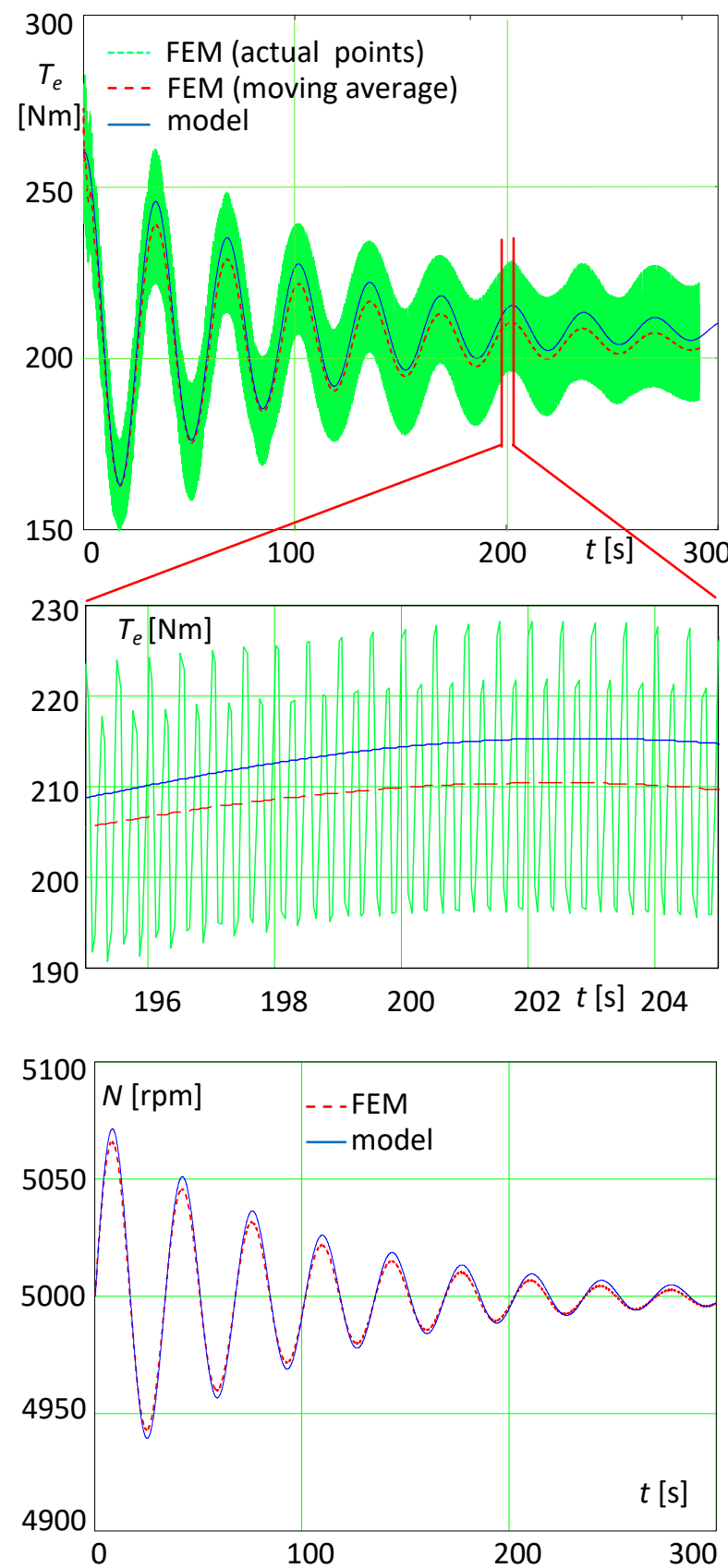

Fig. 8. Comparison between the model results and FEM results as concerns electromagnetic torque $T_{e}$ (top) and mechanical speed $N$ (bottom).

\section{EXAMPLE OF COMPARISON WITH A SIMPLIFIED MODEL}

Once the proposed model has been validated, a comparison is carried out with the torque obtained by a simplified model, which assumes $\psi_{s d}$ dependent only on $i_{s d}$ and $\psi_{s q}$ dependent only on $i_{s q}$ (no cross coupling: subscript NOcc). Thus, two new functions $\psi_{s d_{-} N O c c}$ and $\psi_{s q_{-} N O c c}$ are defined. They can be obtained starting from (4), by expressing $i_{s d}, i_{s q}$ as a function of $I_{s d q}, \varphi_{I}$. In particular, amplitude and phase of $i_{s d}, i_{s q}$ can be expressed as

$\left|i_{s d}\right|=\left|I_{s d q} \cdot \cos \left(\varphi_{I}\right)\right|, \arg \left(i_{s d}\right)=$ if $\left(-\pi / 2<\varphi_{I}<\pi / 2 ; 0 ; \pi\right)$

$\left|i_{s q}\right|=\left|I_{s d q} \cdot \sin \left(\varphi_{I}\right)\right|, \arg \left(i_{s q}\right)=$ if $\left(0<\varphi_{I}<\pi ; \pi / 2 ;-\pi / 2\right)$

Inserting these quantities in (4) yields 
$\psi_{s d \_N O c c}\left(I_{s d q}, \varphi_{I}\right)=$

$\psi_{s d}\left(\left|I_{s d q} \cdot \cos \left(\varphi_{I}\right)\right|\right.$, if $\left.\left(-\pi / 2<\varphi_{I}<\pi / 2 ; 0 ; \pi\right)\right)$

$\psi_{s q_{-} N O c c}\left(I_{s d q}, \varphi_{I}\right)=$

$\psi_{s q}\left(\left|I_{s d q} \cdot \sin \left(\varphi_{I}\right)\right|\right.$, if $\left.\left(0<\varphi_{I}<\pi ; \pi / 2 ;-\pi / 2\right)\right)$

By means of (27), flux linkage and torque of the model without cross coupling can be expressed simply as

$$
\begin{gathered}
\bar{\psi}_{s d q_{-} N O c c}\left(I_{s d q}, \varphi_{I}\right)=\psi_{s d_{-} N O c c}\left(I_{s d q}, \varphi_{I}\right)+j \psi_{s q_{-} N O c c}\left(I_{s d q}, \varphi_{I}\right) \\
T_{e_{-} N O c c}=-2 \cdot n \cdot \operatorname{Im}\left(\bar{\psi}_{s d q_{-} N O c c} \mathrm{i}_{s d q}\right)
\end{gathered}
$$

Fig. 9 shows, for three values of $I_{s d q}$, the torque as a function of $\varphi_{I}$, considering the proposed model (15) (continuous line) and the simplified model (29) (dotted line). It is apparent that the simplified model works well just for low currents, but it works worse and worse as saturation increases.

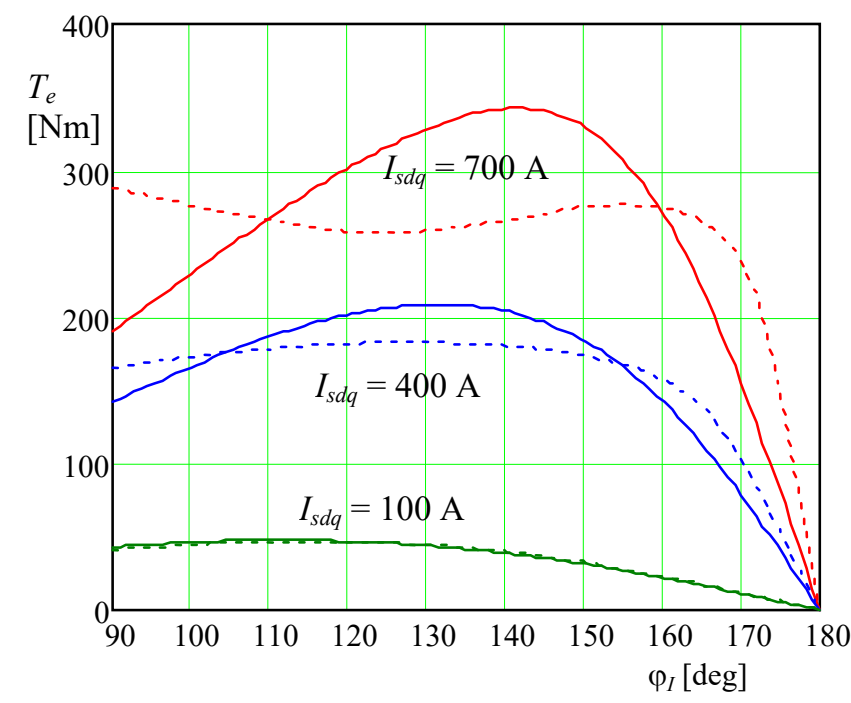

Fig. 9. Torque as a function of $\varphi_{I}$ for three values of $I_{s d q}$, for the proposed model (15) (continuous line) and the simplified model (29) (dotted line).

\section{CONCLUSION}

The paper deals with an IPM machine model able to taking into account both saturation and cross coupling effects accurately. The model does not use inductances, but the direct link flux linkage - current and the inverse link current flux linkage, in terms of space vectors: the procedure adopted to define the cited links is described in detail.

The model has been validated by means of 2D FEM simulations, both for operation in steady state and in transient operating conditions. The model simulation time is greatly reduced compared with FEM simulation time, thus representing a useful tool for design and operation analysis.
If the torque is evaluated by a simplified model which does not take into account cross coupling, a great error occurs at high currents.

\section{REFERENCES}

[1] B. Stumberger, G. Stumberger, D. Dolinar, A. Hamler and M. Trlep, "Evaluation of saturation and cross-magnetization effects in interior permanent-magnet synchronous motor," in IEEE Transactions on Industry Applications, vol. 39, no. 5, pp. 1264-1271, Sept.-Oct. 2003. doi: 10.1109/TIA.2003.816538.

[2] Y. Li, Z. Q. Zhu, D. Howe and C. M. Bingham, "Modeling of CrossCoupling Magnetic Saturation in Signal-Injection-Based Sensorless Control of Permanent-Magnet Brushless AC Motors," in IEEE Transactions on Magnetics, vol. 43, no. 6, pp. 2552-2554, June 2007. doi: 10.1109/TMAG.2007.892319.

[3] Z. Q. Zhu, Y. Li, D. Howe, C. M. Bingham and D. Stone, "Influence of Machine Topology and Cross-Coupling Magnetic Saturation on Rotor Position Estimation Accuracy in Extended Back-EMF Based Sensorless PM Brushless AC Drives," 2007 IEEE Industry Applications Annual Meeting, New Orleans, LA, 2007, pp. 2378-2385. doi: 10.1109/07IAS.2007.359.

[4] A. Wang, H. Li and C. Liu, "Assessments of magnetic cross-coupling impacts on interior permanent magnet machine controls for electric vehicles," 2009 International Conference on Power Electronics and Drive Systems (PEDS), Taipei, 2009, pp. 1435-1438. doi: 10.1109/PEDS.2009.5385821.

[5] H. Nagura, Y. Iwaji, J. Nakatsugawa and N. Iwasaki, "New vector controller for PM motors which modeled the cross-coupling magnetic flux saturation," The 2010 International Power Electronics Conference - ECCE ASIA -, Sapporo, 2010, pp. 1064-1070. doi: 10.1109/IPEC.2010.5542095

[6] Z. Li and H. Li, "MTPA control of PMSM system considering saturation and cross-coupling," 2012 15th International Conference on Electrical Machines and Systems (ICEMS), Sapporo, 2012, pp. 1-5.

[7] R. Bojoi, A. Cavagnino, M. Cossale and S. Vaschetto, "Methodology for the IPM motor magnetic model computation based on finite element analysis," IECON 2014 - 40th Annual Conference of the IEEE Industrial Electronics Society, Dallas, TX, 2014, pp. 722-728. doi: 10.1109/IECON.2014.7048580

[8] A. Rabiei, T. Thiringer, M. Alatalo and E. A. Grunditz, "Improved Maximum-Torque-Per-Ampere Algorithm Accounting for Core Saturation, Cross-Coupling Effect, and Temperature for a PMSM Intended for Vehicular Applications," in IEEE Transactions on Transportation Electrification, vol. 2, no. 2, pp. 150-159, June 2016. doi: 10.1109/TTE.2016.2528505

\section{BIOGRAPHIES}

Antonino Di Gerlando received his MS degree in electrical engineering from the Politecnico di Milano, Italy, in 1981. Currently, he is a Full Professor at the Department of Energy at Politecnico di Milano. Fields of interest: design and modeling of electrical machines, converters and drive systems. He is a senior member of IEEE and a member of the Italian Association of the Electric and Electronic Engineers (AEI).

Giovanni Maria Foglia received his MS degree and the $\mathrm{PhD}$ in electrical engineering at Politecnico di Milano, Milano, Italy, in 1997 and 2000.

Currently, he is an Associate Professor at the Department of Energy at Politecnico di Milano, and his main field of interest is the analysis and design of PM electrical machines.

Roberto Perini (M'10) received his MS degree and the $\mathrm{PhD}$ in electrical engineering from the Politecnico di Milano, Milano, Italy.

Currently, he is an Associate Professor at the Department of Energy at Politecnico di Milano. His interests are in the design and modeling of electrical machines and power electronics. 